\title{
Role of substrate recognition in modulating strigolactone receptor selectivity in witchweed
}

Received for publication, May 21, 2021, and in revised form, July 26, 2021 Published, Papers in Press, August 24, 2021,

https://doi.org/10.1016/j.jbc.2021.101092

\section{Jiming Chen ${ }^{1}$, Alexandra White ${ }^{2}$, David C. Nelson ${ }^{2}{ }^{-1}$, and Diwakar Shukla $1,3,4,5,6, *$ (D)}

From the ${ }^{1}$ Department of Chemical and Biomolecular Engineering, University of Illinois at Urbana-Champaign, Urbana, Illinois, USA; ${ }^{2}$ Department of Botany and Plant Sciences, University of California, Riverside, Riverside, California, USA; ${ }^{3}$ Center for Biophysics and Quantitative Biology, ${ }^{4}$ National Center for Supercomputing Applications, ${ }^{5}$ Beckman Institute for Advanced Science and Technology, and ${ }^{6} \mathrm{NIH}$ Center for Macromolecular Modeling and Bioinformatics, University of Illinois at UrbanaChampaign, Urbana, Illinois, USA

Edited by Wolfgang Peti

Witchweed, or Striga hermonthica, is a parasitic weed that destroys billions of dollars' worth of crops globally every year. Its germination is stimulated by strigolactones exuded by its host plants. Despite high sequence, structure, and ligandbinding site conservation across different plant species, one strigolactone receptor in witchweed, ShHTL7, uniquely exhibits a picomolar EC50 for downstream signaling. Previous biochemical and structural analyses have hypothesized that this unique ligand sensitivity can be attributed to a large binding pocket volume in ShHTL7 resulting in enhanced ability to bind substrates, but additional structural details of the substrate-binding process would help explain its role in modulating the ligand selectivity. Using long-timescale molecular dynamics simulations, we demonstrate that mutations at the entrance of the binding pocket facilitate a more direct ligand-binding pathway to ShHTL7, whereas hydrophobicity at the binding pocket entrance results in a stable "anchored" state. We also demonstrate that several residues on the D-loop of $A t \mathrm{D} 14$ stabilize catalytically inactive conformations. Finally, we show that strigolactone selectivity is not modulated by binding pocket volume. Our results indicate that while ligand binding is not the sole modulator of strigolactone receptor selectivity, it is a significant contributing factor. These results can be used to inform the design of selective antagonists for strigolactone receptors in witchweed.

Strigolactones are a class of plant hormones responsible for regulating shoot branching and root architecture in plants (1-4). They have also been found to induce seed germination in the parasitic Striga genus (5). Estimates of global crop losses due to Striga parasites are in excess of $\$ 10$ billion per year, warranting a need for effective Striga control (6). Strigolactone perception is controlled by a family of proteins called DWARF14, which possess a conserved $\alpha-\beta$ hydrolase fold with a hydrophobic cavity in which the substrate binds. D14 and its closely related homolog KAI2 contain a strictly conserved SerHis-Asp catalytic triad (Fig. 1A). Strigolactone signaling

* For correspondence: Diwakar Shukla, diwakar@illinois.edu. responses are believed to be dependent on enzymatic hydrolysis of the substrate and subsequent covalent modification of the enzyme by a hydrolysis intermediate, which remains covalently bound to catalytic residues during the subsequent signaling step (7-10). Following hydrolysis, the receptor undergoes a large conformational change that enables it to associate with MAX2 and SMXL proteins, which are then ubiquitinated and degraded by the proteasome (11). Recent evidence has also suggested that signal can be transduced by intact strigolactone molecules (12) and that MAX2 proteins may act as a repressor of strigolactone hydrolysis (13).

Despite 44\% sequence identity between Arabidopsis thaliana and Striga hermonthica strigolactone receptors, 78\% sequence similarity, and a highly conserved structure between different species (Fig. 1C), one receptor in S. hermonthica, ShHTL7 uniquely exhibits a picomolar range EC50 for downstream signaling for inducing a germination response, compared with micromolar ranges for other strigolactone receptors (14). An evolutionary analysis by Conn et al. (15) revealed that ShHTL proteins evolved the ability to perceive strigolactone via convergent evolution. ShHTL proteins are paralogs of KAI2 proteins, which perceive seed germination stimulants in plants and evolved strigolactone sensitivity independently of D14 proteins (15). KAI2 proteins are generally grouped into three clades, the KAI2c (conserved) clade, which is the most KAI2-like and has sensitivity to karrikins but not strigolactones, the KAI2i intermediate clade, and the divergent KAI2d clade that is strigolactone-sensitive but not karrikin-sensitive. Further studies have hypothesized that the high strigolactone sensitivity found in several members of the divergent clade of ShHTL proteins, which includes ShHTL7, can be attributed to their larger binding pocket volume compared with other members of the D14/KAI2 superfamily of proteins $(14,16)$. Additionally, an isothermal titration calorimetry and crystallography study by Bürger et al. (17) suggested that the T2-T3 loop of KAI2 proteins is able to modulate pocket size, which in turn is able to influence binding affinity. However, this hypothesis relies on pocket volumes computed from crystal structures, which can only provide static "snapshots" of the protein. In an aqueous 
A

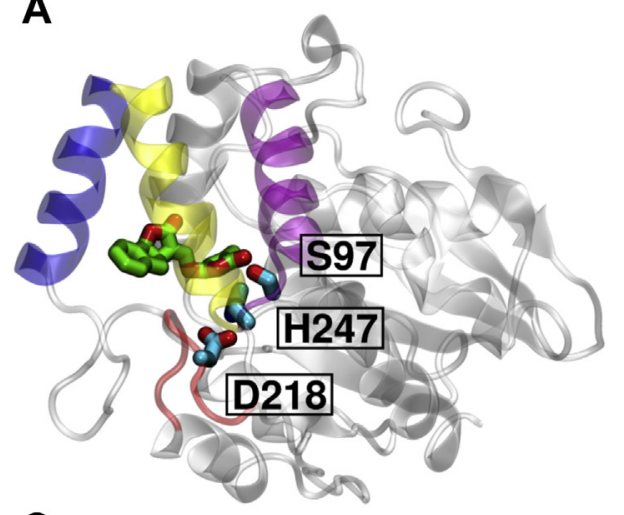

C

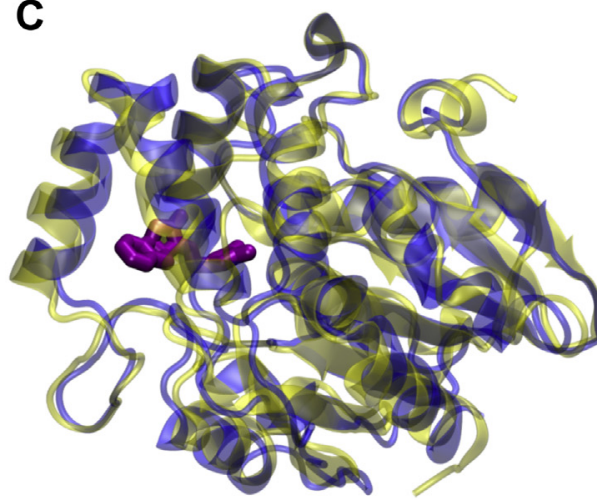

B
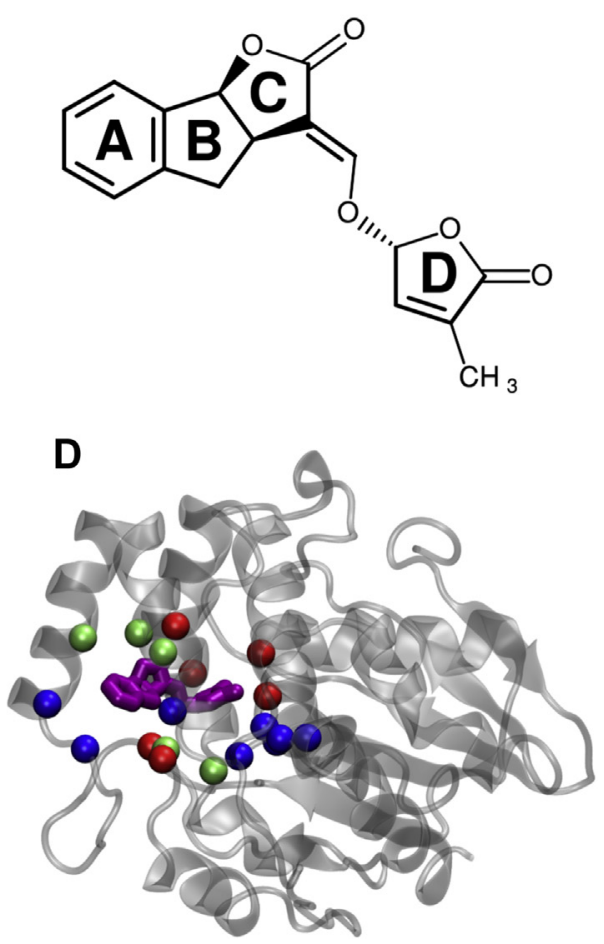

Figure 1. Structures of the strigolactone receptor and synthetic strigolactone analog GR24. $A$, structure of Arabidopsis thaliana strigolactone receptor AtD14 in complex with strigolactone analog GR24 (green). The T1, T2, and T3 helices are shown in blue, yellow, and purple, respectively, and the D-loop is shown in red. The serine-histidine-aspartate catalytic triad is shown in cyan. $B$, structure of synthetic strigolactone analog GR24 ${ }^{5 D S}$, which possesses the stereochemistry of naturally occuring strigolactones. C, structural alignment of AtD14 (blue) and ShHTL7 (yellow). Bound GR24 is shown in purple. D, similarity of binding pocket residues. Blue spheres indicate conserved residues between AtD14 and ShHTL7, red spheres indicate similar residues, and green spheres indicate different residues. Full sequence and secondary structure alignments are shown in Figure S1.

environment, the pocket volume is likely to fluctuate due to conformational flexibility of the protein.

Alternatively, differences in the substrate binding mechanism can contribute to enhanced signaling ability in one protein over the other. Differences in the substrate-binding process can enhance signaling in two ways: (i) A higher binding affinity for the ligand can increase the residence time of the ligand in the pocket, leading to increased probability of enzymatic hydrolysis occurring, or (ii) a lower free energy barrier of binding can enhance the rate of binding, thus enhancing the apparent rates of subsequent steps. Characterizing the role of these effects in producing the uniquely high sensitivity of ShHTL7 requires a detailed structural and dynamical characterization of the binding process. While structures of the protein-ligand complexes could provide insights into differences in binding affinity, mechanistic details of the binding process can additionally determine the effects of sequence differences in residues outside the binding pocket on ligand binding. The only currently available crystal structure of a strigolactone-bound D14 protein is a structure of OsD14, the Oryza sativa ortholog of AtD14 ( 74\% sequence identity), bound to GR24 (18). There is uncertainty surrounding the accuracy of this as well as other strigolactone receptor crystal structures bound to various ligands due to low electron density of ligands within the binding pocket (19), and there is an inherent lack of dynamical information contained in crystal structures. Making direct biophysical measurements on the binding process is also particularly challenging for strigolactone receptors since it is known to hydrolyze its ligand. This coupling of binding and hydrolysis makes it difficult to elucidate the effects of substrate binding on signaling independently of subsequent steps. A powerful method that can be used to characterize the strigolactone binding process is molecular dynamics (MD) simulations (20-26). When used with Markov state models (MSMs), simulations can provide detailed kinetic and thermodynamic information about ligandbinding processes at atomic-level resolution (27-32). Furthermore, MSMs allow us to perform analysis on a large number of short simulations rather than a single long simulation $(33,34)$, which greatly decreases the time required to obtain sufficient data. MD simulations have previously been used to characterize other conformational dynamics and substrate binding in other plant proteins (20,21, 24-26).

Recently, Hu et al. (17) employed biased MD simulations to characterize the mechanism of the smoke-derived compound $\mathrm{KAR}_{1}$ to $A t \mathrm{KAI} 2$, a homolog of $A t \mathrm{D} 14 \quad(\sim 50 \%$ sequence identity). However, a limitation to this study is the biasing methods that were used, which have an inherent assumption that the ligand can only bind in a single binding pose and via a single pathway. Here, we employ long timescale $(\sim 400 \mu \mathrm{s}$ 
aggregate) unbiased MD simulations, allowing for a highresolution, dynamical view of the substrate recognition mechanisms in AtD14 and ShHTL7. We demonstrate that ShHTL7 is more efficient at binding GR24 and is also more effective at positioning GR24 for hydrolysis than $A t D 14$. Additionally, we show that while differences in the ligandbinding process do contribute to the high ligand sensitivity in ShHTL7, these differences are not caused by the difference in the crystal structure pocket volume.

\section{Results}

\section{Free energy profile of the binding process}

Using $\sim 200 \mu$ s aggregate of MD simulations each on GR24 binding to AtD14 and ShHTL7, we computed the free energy landscapes of the complete ligand-binding processes (Fig. 2). These landscapes were projected onto A-ring-catalytic serine distance and D-ring-catalytic serine distance for the purpose of distinguishing different binding modes of the ligand. Free energy minima discernable from these landscapes are the bound state $(\alpha)$, consistent with the crystal structure of GR24bound OsD14 (PDB 5DJ5) (18); an inverse bound state ( $\beta$ ), and an "anchored" intermediate state $(\gamma)$. The most stable minimum for both proteins corresponds to the bound state with the butenolide ring of the ligand oriented into the binding pocket and close to S97/95 of the catalytic triad. Both AtD14 and ShHTL7 are also capable of binding GR24 in an inverse pose, in which the A-ring is oriented into the pocket and the butenolide ring (D-ring) is oriented toward the pocket entrance. The canonical model of strigolactone signaling involves a catalytic mechanism in which S97/95 nucleophilically attacks the ligand upon the D-ring $(7,8)$, indicating that this inverse-bound pose is likely catalytically inactive and thus signaling incompetent.

Using the method in Buch et al. (27), we calculated the free energy for GR24 binding to be $-5.5 \mathrm{kcal} / \mathrm{mol}$ in AtD14 and $5.7 \mathrm{kcal} / \mathrm{mol}$ in $S h H T L 7$. Uncertainty calculations for standard binding free energies are detailed in Tables S8 and S9. Free energy landscapes with respect to the slowest motions in the binding process are shown in Figure S2. A previously reported dissociation constant $\left(K_{d}\right)$ for GR24 binding to AtD14 based on an isothermal titration calorimetry measurement is $0.30 \pm$ $0.02 \mu \mathrm{M}$, which corresponds to a free energy of $-8.7 \mathrm{kcal} / \mathrm{mol}$ at the experimental temperature of $293 \mathrm{~K}$ (35). These free energy values were computed using the equation $\Delta G=-$ $R T \ln K_{d}$, where $T$ is the temperature at experimental conditions and $K_{d}$ is the reported dissociation constant. However, this value is likely the free energy associated with both binding and hydrolysis because a significant positive entropy change $(+19.5 \mathrm{cal} / \mathrm{mol}$ * $\mathrm{K})$ upon binding is reported. Ligand binding is expected to have a negative entropy change associated with loss of configurational entropy of the ligand, but a hydrolysis reaction in which GR24 is split into its ABC-ring and D-ring would more likely yield a positive entropy change. The $K_{d}$ for GR24 binding to ShHTL7 is estimated to be $0.92 \pm 0.01 \mu \mathrm{M}$ based on microscale thermophoresis assay and $0.39 \pm 0.05 \mu \mathrm{M}$ based on a tryptophan fluorescence assay, which correspond to a free energies of $-8.7 \mathrm{kcal} / \mathrm{mol}$ and $-8.2 \mathrm{kcal} / \mathrm{mol}$, respectively, at $298 \mathrm{~K}(36)$.

\section{Hydrophobic to polar sequence variations at pocket entrance destabilize anchored-intermediate state in ShHTL7}

A notable difference in the binding pathways is the stability of the "anchored" intermediate state $(\gamma)$. Based on the free energy landscapes, the anchored intermediate state is $\sim 1.5-2 \mathrm{kcal} / \mathrm{mol}$ more stable in relation to the bound minimum in AtD14 than in ShHTL7. This indicates that the ligand is more likely to interact with the pocket entrance during the binding process in AtD14 than in ShHTL7. To further investigate the pocket entrance-anchoring observed in $A t \mathrm{D} 14$, we computed per-residue ligand contact probabilities for both AtD14 and ShHTL7 (Fig. 3, $A$ and $B$ ). In agreement with the free energy landscapes of the binding process, the regions of highest ligand contact probabilities in both proteins were the interior of the binding pocket. Additionally, the region directly outside the binding pocket shows considerably higher ligand contact probability in AtD14 than in ShHTL7. This further indicates the presence of a stable interaction between the
A

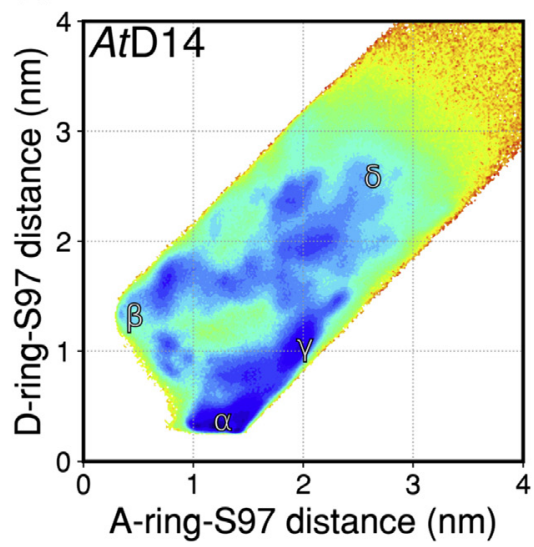

B

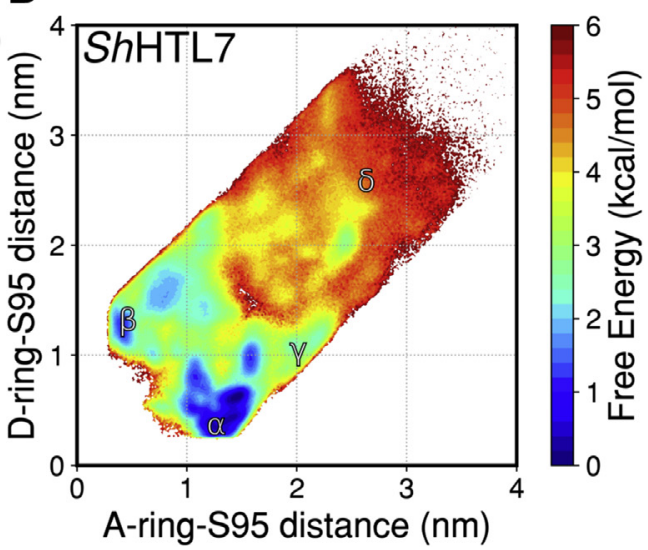

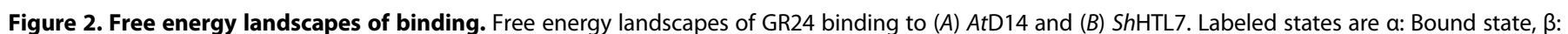
Inverse bound state, $\gamma$ : Anchored state, $\delta$ : Unbound states 


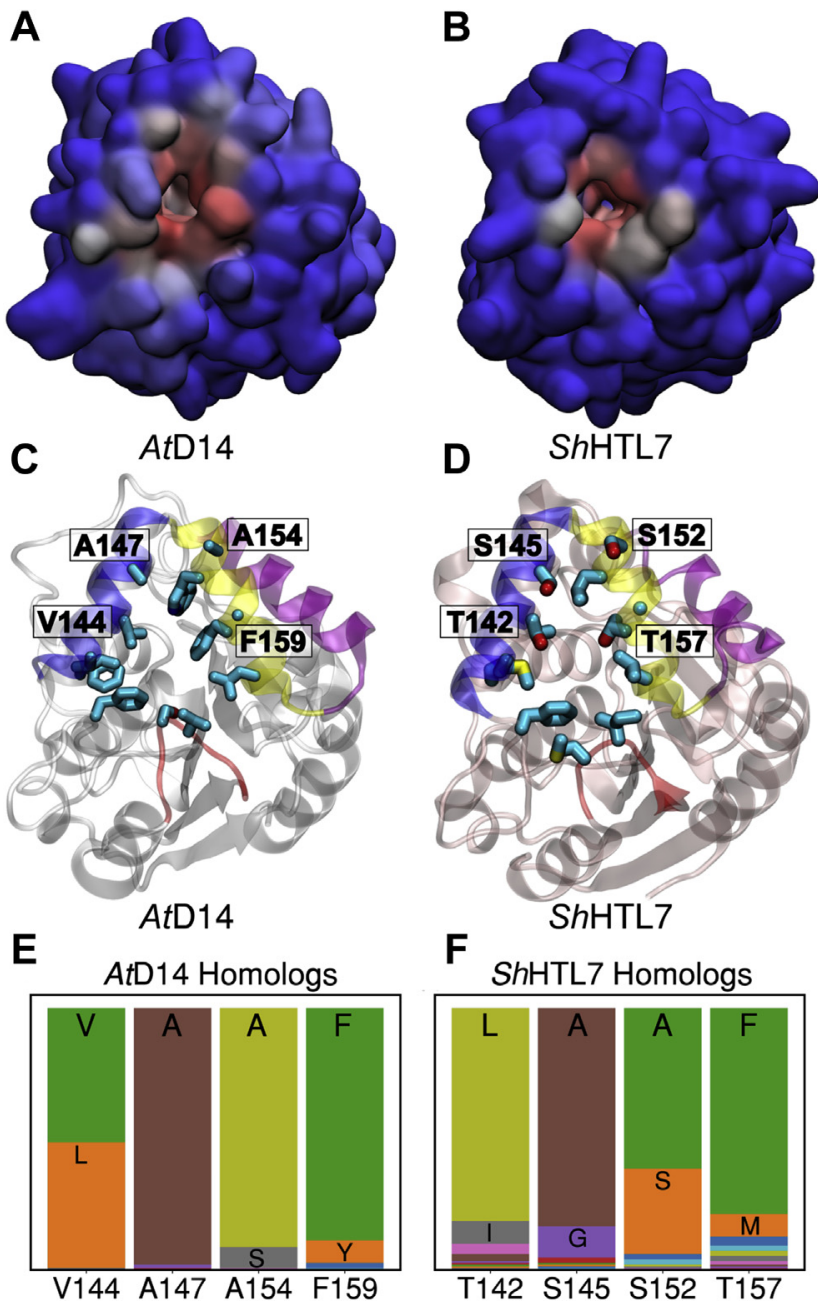

Figure 3. Ligand contact with pocket entrance. Residue-ligand contact probabilities for $(A) A t D 14$ and $(B)$ ShHTL7. Red regions indicate high ligand contact probability, and blue regions indicate low ligand contact probability. C, AtD14 pocket entrance residues in contact with the ligand in the anchored state and $(D)$ corresponding residues in ShHTL7. Hydrophobic to polar substitutions are labeled. $E$, most frequently occurring residues in pocket entrance sites for AtD14 homologs and (F) ShHTL7 homologs. Residue lists for each site can be found in Table S2.

ligand and a patch of $\mathrm{T} 1$ and $\mathrm{T} 2$ helix residues outside the binding pocket. A comparison of residues in contact with the ligand in the $A t \mathrm{D} 14$ anchored state and their corresponding residues in ShHTL7 is shown in Figure 3, $C$ and $D$. Four residues on the $\mathrm{T} 1$ and $\mathrm{T} 2$ helices are mutated from hydrophobic to polar residues between AtD14 and ShHTL7: V144 (T142), A147 (S145), A154 (S152), and F159 (T157). Increased polarity at the pocket entrance prevents stable hydrophobic interactions from forming with the ABC rings of GR24. Since interactions between GR24 and the pocket entrance are largely hydrophobic, these mutations are likely to destabilize the anchored state in ShHTL7, thus potentially leading to enhanced binding kinetics. This is consistent with the observation that a G158E mutant of AtD14 displayed increased hydrolytic activity toward GR24 despite becoming signaling inactive (7). Additionally, in a recent study introducing a femtomolar-range suicide germination compound for Striga, several mutations at the binding pocket entrance resulted in an increase in IC50 for competitive binding of the compound to SPL7 (37). While this is not directly comparable since the measurements were done with a different ligand, it nonetheless supports the hypothesis that residues at the pocket entrance play an important role in ligand binding. Using the ConSurf server (38), we also computed the site conservation of these four residues among homologs of AtD14 and ShHTL7. Most frequent residues occupying the four pocket entrance sites among AtD14 and ShHTL7 are shown in Figure 3, E and $F$. The four sites show high conservation among both sets of homologs. However, while the pocket entrance residues in AtD14 all match the most frequent residues of the given sites, the pocket entrance residues in ShHTL7 are all less common substitutions. Notably, the most common residues at pocket entrance sites in ShHTL7 homologs are hydrophobic, as in $A t \mathrm{D} 14$, indicating that polarity at the pocket entrance is not a common feature even among close homologs of ShHTL7. Parameters for the ConSurf calculation can be found in Table S1, and conservation scores and residue lists can be found in Table S2.

\section{Catalytically competent D-loop conformation more stable in ShHTL7 than in AtD14}

Enzymatic hydrolysis of the substrate requires the D-loop of the protein to be in a D-in conformation, in which there is interaction between the aspartate (D218/217) and histidine (H247/246) of the catalytic triad. This is known due to previous mutagenesis experiments that have shown elimination of enzymatic activity upon mutation of any of the catalytic triad residues $(7,12,39,40)$. We computed free energy profiles of the binding process projected onto catalytic D-catalytic $\mathrm{H}$ distance and ligand-pocket distance (Fig. 4). The catalytically active state in which D218/217 is oriented into the binding pocket (D-in) and the substrate is bound is most stable in ShHTL7. However, AtD14 exhibits highly stable conformations in which the D218 is oriented away from H247, rendering the protein catalytically inactive. The D-out conformations in $A t \mathrm{D} 14$ are $\sim 3-5 \mathrm{kcal} / \mathrm{mol}$ more stable than in ShHTL7, indicating the presence of stabilizing interactions that facilitate the formation of these catalytically incompetent conformations. Upon comparison of the D-loop sequences in AtD14 (AKDVSVPA) and ShHTL7 (SNDIMVPV), we identified three sequence variations with differing residue types (i.e., hydrophobic to hydrophilic, charged to neutral): A216S, $\mathrm{K} 217 \mathrm{~N}$, and S220M. Based on free energy profiles of key contacts involving these residues, we determine that each of these three mutations contributes to stabilization of the D-out conformation in AtD14 (Fig. 5).

The A216S sequence variation is located on the end of the D-loop closest to the T2 helix. The corresponding residue in ShHTL7 is S215, which is able to form hydrogen bonding interactions with the adjoining $\beta$-strand. This limits the range of motion of the D-loop. The A216 residue in AtD14 is unable to form a hydrogen bond with the adjacent $\beta$-strand, allowing for increased D-loop motion. Additionally, the free energy landscape indicates that less stable D218-H247 interaction is 
A

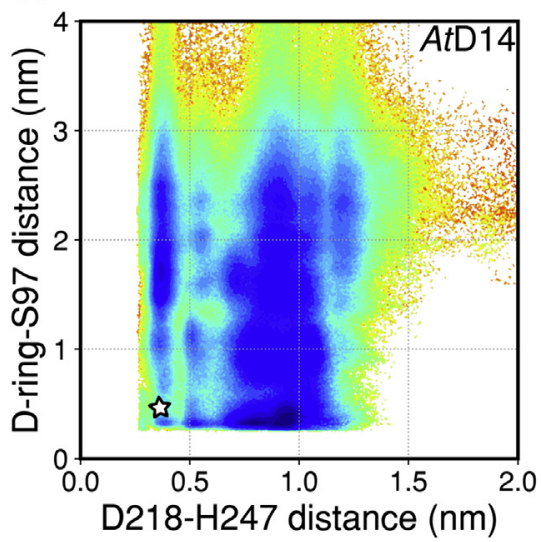

B

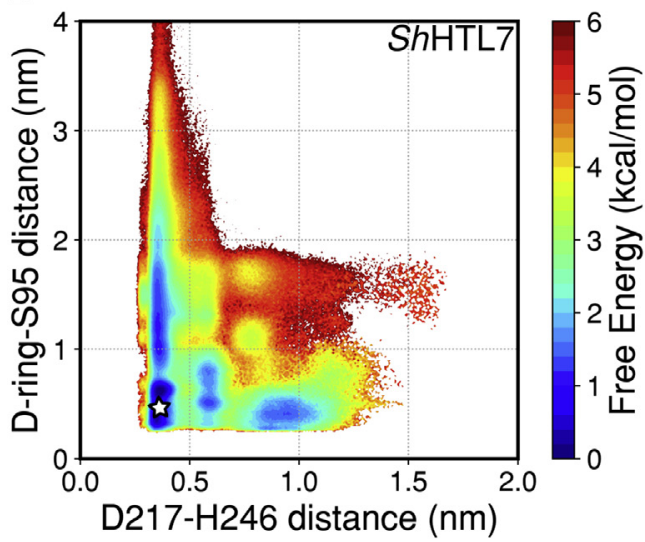

Figure 4. Free energy landscape of D-loop conformation. Free energy landscapes of GR24 binding to (A) AtD14 and (B) ShHTL7 projected onto D218/217$\mathrm{H} 247 / 246$ distance and ligand-S97/95 distance. The star demarcates the catalytically active state in which the ligand is bound and the aspartate and histidine of the catalytic triad are in contact.

observed in the absence of A216-backbone interaction (Fig. 5A). This implies that the interaction between S215 in ShHTL7 and the adjacent backbone helps to stabilize the D-loop in the catalytically active D-in conformation.
The K217 residue in AtD14 can form salt bridges with nearby negatively charged residues (D167, E245). In ShHTL7, the corresponding residue is N216, which eliminates a positive charge and prevents the formation of stable salt bridges. In
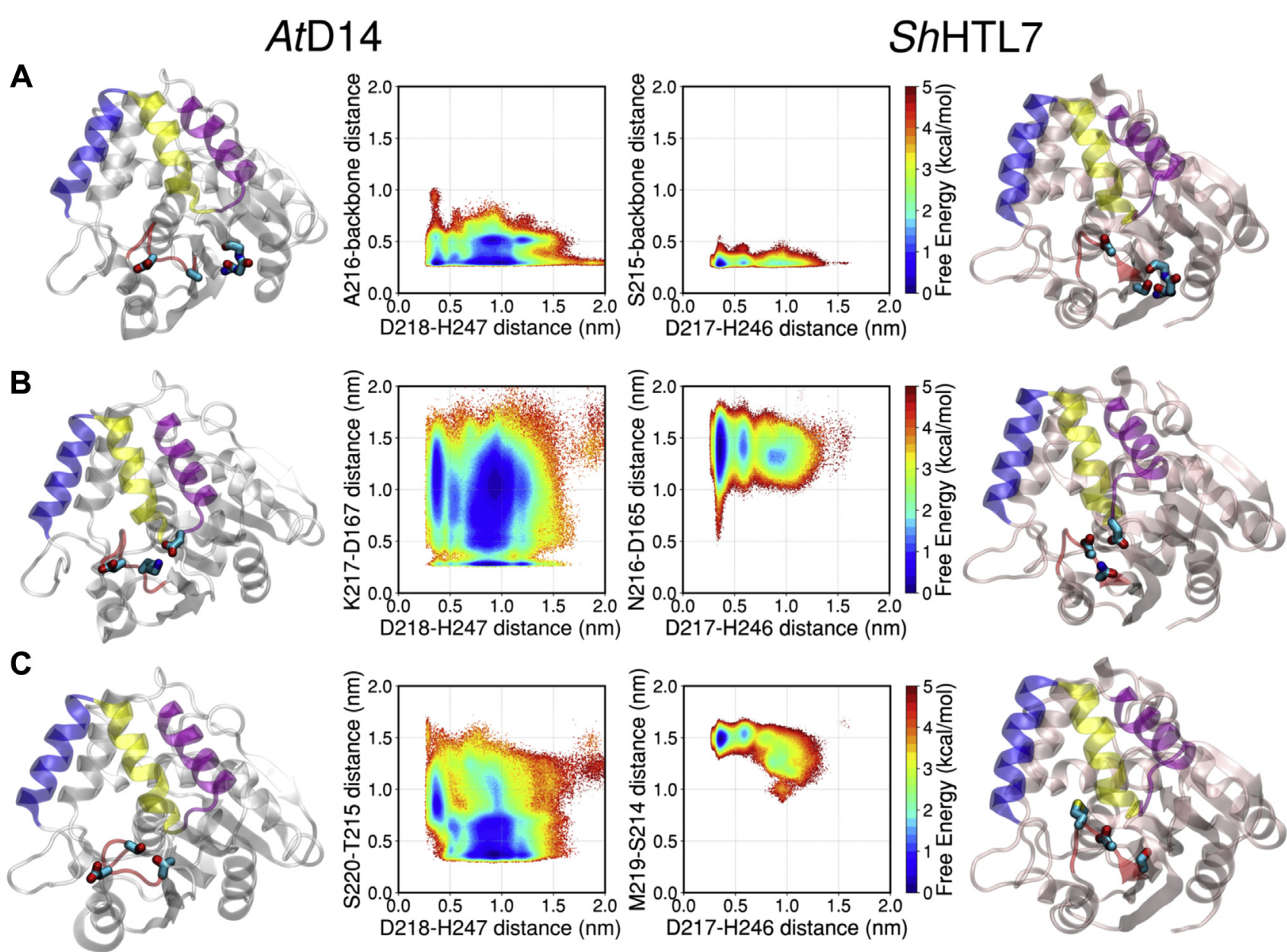

Figure 5. Contacts stabilizing the D-out conformation relative to the D-in conformation in AtD14. The D-in conformation is defined as having an interaction between D218/217 and H247/246, which means that the D-H distance is within $\sim 0.5 \mathrm{~nm}$. A, the S215-backbone interaction (S215-backbone distance $<0.5 \mathrm{~nm}$ ) in ShHTL7 is lost in AtD14. B, the K217-D167 salt bridge in AtD14 (K217-D167 distance < 0.5 nm) is lost in ShHTL7. C, the S220-T215 hydrogen bond in AtD14 (S220-T215 distance $<0.5 \mathrm{~nm}$ ) is lost in ShHTL7. 
particular, formation of the K217-E245 salt bridge in AtD14 destabilizes the D218-H247 interaction, whereas absence of this salt bridge in ShHTL7 allows for a stable D217-H246 interaction (Fig. 5B). In addition to D167, K217 can also form a salt bridge with E245. However, equally stable D218-H247 interactions are observed both in the presence and absence of the K217-E245 salt bridge, which indicates that this interaction does not destabilize the D218-H247 interaction (Fig. S3). In ShHTL7, N216 is able to form a hydrogen bond with E244. As observed in AtD14, the D217-H246 interaction remains intact both in the presence and absence of the N216-E244 hydrogen bond (Fig. S3).

Finally, S220 in AtD14 can form a hydrogen bond with T215, which locks D218 in an outward-oriented position. The free energy landscape with respect to D218-H247 distance and S220-T215 distance indicates that D218-H247 contact is nearly eliminated in the presence of the S220T215 hydrogen bond (Fig. 5C). The corresponding residue to S220 in ShHTL7 is M219, which is hydrophobic and thus unable to form a hydrogen bond with S214, the corresponding ShHTL7 residue to T215 in AtD14. In the absence of an M219-S214 hydrogen bond, a stable D217-H246 interaction is observed.

\section{Large fluctuations in AtD14 pocket volume facilitate binding-incapable states and nonproductive binding}

Previous structural studies have hypothesized that a large binding pocket volume in ShHTL7 is responsible for its uniquely high affinity for strigolactones (14, 16, 41). To evaluate this hypothesis, we computed the probability distributions of pocket volumes in AtD14 and ShHTL7 over the course of our simulations (Fig. 6). The average pocket volumes of the two proteins are in close agreement with each other ( $\mu=268 \AA^{3}$ and $274 \AA^{3}$ for AtD14 and ShHTL7, respectively). However, AtD14 displays a significantly broader distribution of binding pocket volumes $(\sigma=$ $90 \AA^{3}$ and $45 \AA^{3}$ for $A t \mathrm{D} 14$ and ShHTL7, respectively). Using the same pocket volume calculation metrics, we computed the pocket volumes of the apo crystal structures of AtD14 and ShHTL7 to be $215 \AA^{3}$ and $358 \AA^{3}$, respectively. While the ShHTL7 crystal structure does have a larger binding pocket volume than the AtD14 crystal structure, this difference in pocket volume decreases significantly in an aqueous environment. In both proteins, the primary modulator of binding pocket volume is a hinging motion between the T1 and T2 helices (Fig. S4). In the lowest-volume states, the binding pocket becomes solvent-inaccessible, rendering the protein unable to bind ligand. The highest-volume states allow for a large ensemble of ligand binding poses to form including many nonproductive binding states in which the ligand is inside the pocket but not positioned for hydrolysis. These results indicate that the decreased tendency of ShHTL7 to change its pocket volume may serve to increase its catalytic efficiency by retaining the pocket in a solvent-accessible conformation while also decreasing the stability of nonproductive binding poses.
A

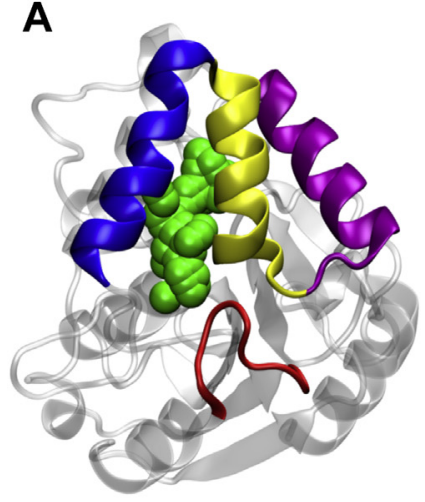

D

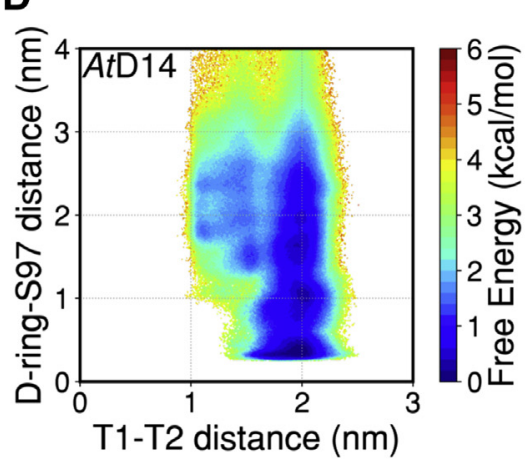

B

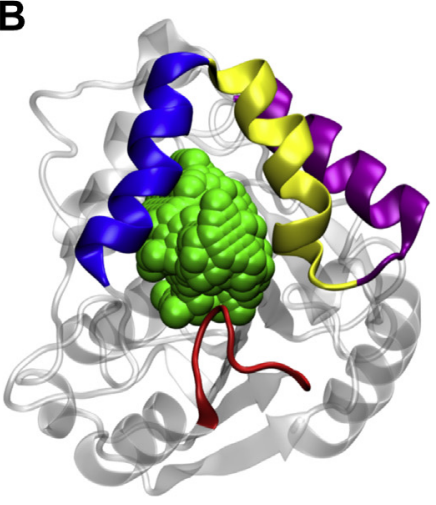

C

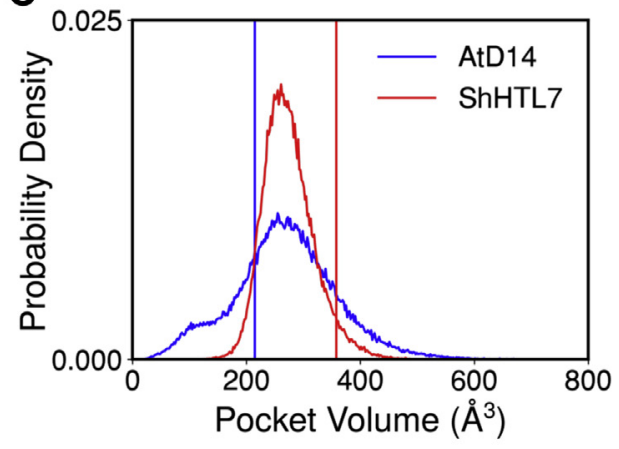

E

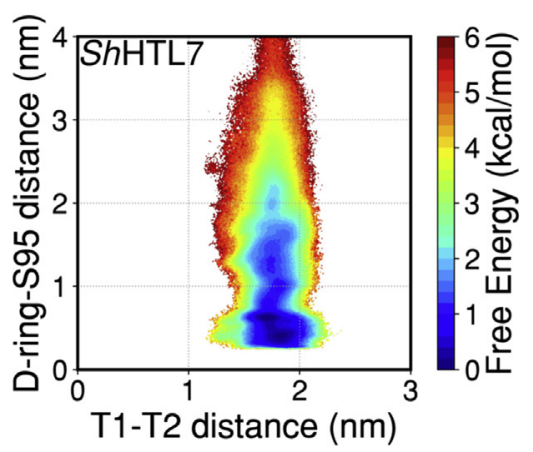

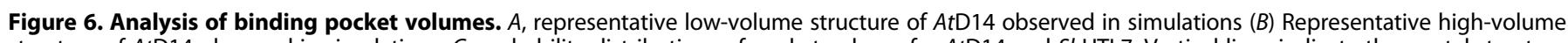

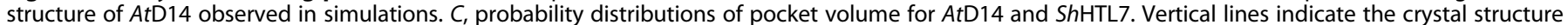

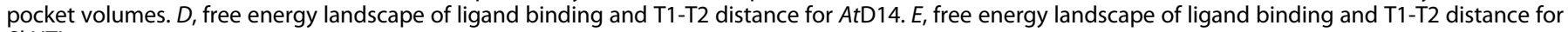
ShHTL7. 
The low-volume states accessible by AtD14 are stabilized by hydrophobic interactions between the $\mathrm{T} 1$ and $\mathrm{T} 2$ helices, indicating that polarity in this region may play a dual role in modulating ligand selectivity. As previously stated, these residues enable a stable intermediate state to form, which acts as a barrier to binding. The low-volume states in which the pocket is nearly solvent inaccessible also show hydrophobic interactions between $\mathrm{T} 1$ and $\mathrm{T} 2$ helix residues, which indicates that these interactions could play a role in stabilizing low-volume states as well. In addition to the hydrophobic contacts between the T1 and T2 helices, a nonconserved salt bridge between the T1 and T4 helices in AtD14 provides stabilization to the low-volume states as well (Fig. 7). Both AtD14 and ShHTL7 have a conserved arginine on the T4 helix (R192/191) that are able to form a salt bridge with E142/E140 on the T1 helix. However, AtD14 also has a second negative residue, E138, on T1 that can form a salt bridge with R192. The free energy landscape of this interaction and the T1-T2 distance indicates that in the presence of this E138-R192 salt bridge, low-volume states are stabilized. This residue is mutated to Q136 in ShHTL7, so a salt bridge cannot form and stabilize low-volume states of the protein.

\section{Discussion}

Using extensive MD simulations, we have characterized the mechanism of substrate binding to strigolactone receptors in full atomistic detail and identified several key differences in the binding mechanisms that contribute to the ligand selectivity between strigolactone receptors. Based on our simulations, GR24 binds to both AtD14 and ShHTL7 in the same binding pose as the reported crystal structure of OsD14-GR24 complex (18). Additionally, since our simulations were performed in an unbiased manner, we were also able to identify several nonproductive bound states in which the ligand is bound but improperly positioned for hydrolysis.

In addition to characterizing the possible ligand poses within the receptor binding pockets, we identified a key anchored intermediate along the binding pathway that is $\sim 1.5 \mathrm{kcal} / \mathrm{mol}$ more stable in $A t D 14$ than in ShHTL7. This difference in stability indicates that the anchored state
A

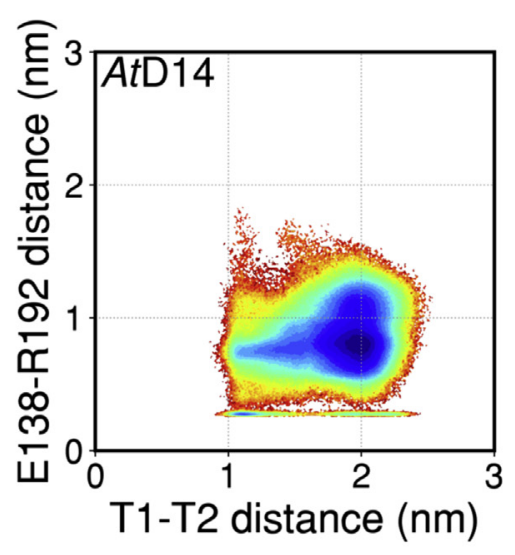

C

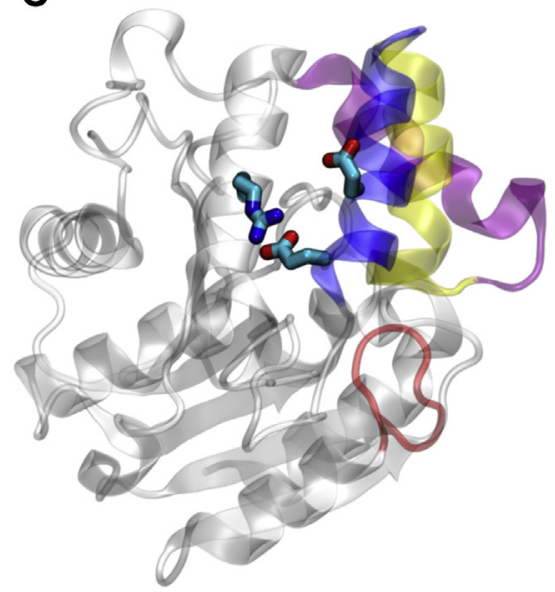

B

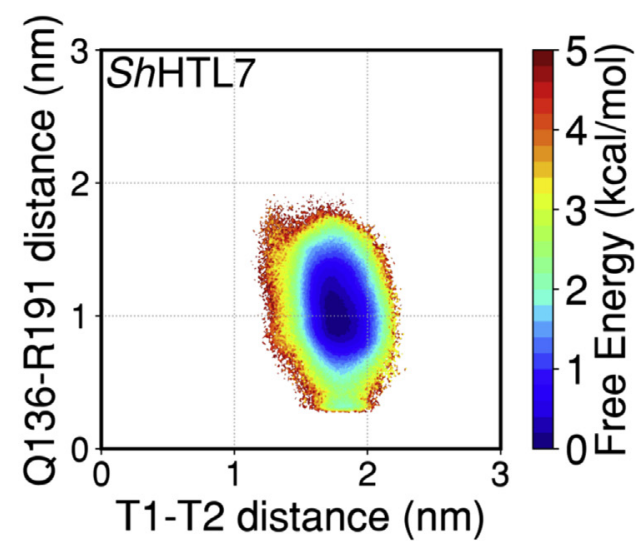

D

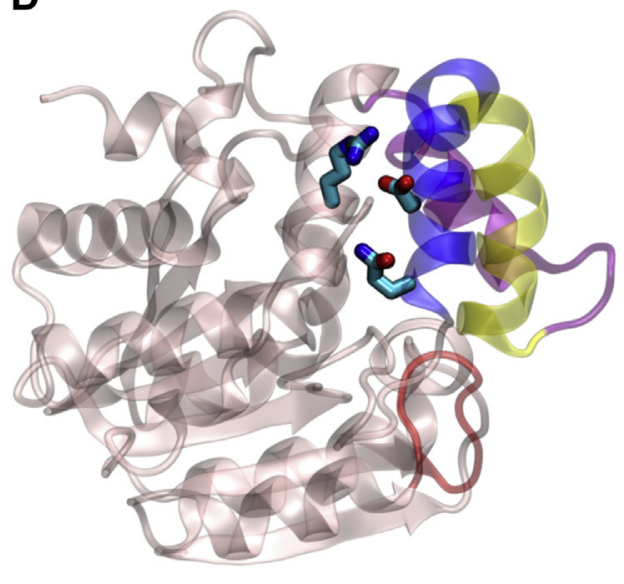

Figure 7. Additional salt bridge in AtD14, which stabilizes the low-volume states. $A$, free energy landscape showing stabilization of low-volume states in the presence of the E138-R192 salt bridge in AtD14, (B) ShHTL7 free energy landscape showing little Q136-R191 interaction as well as lower population of low-volume states than in AtD14. C, E138-R192 salt bridge in AtD14. E142, which can also form a salt bridge with R192, is also shown. D, ShHTL7 salt bridge between R191 and D140, the corresponding residue to E138 in AtD14. Q136, the corresponding residue to E138 in AtD14, is also shown. 
population relative to the bound state is $\sim 12$ times higher in AtD14 than in ShHTL7. This likely results in faster binding kinetics in ShHTL7 than in AtD14, which in turn would lead to a higher observed catalytic turnover rate. Since hydrolysis is driver of receptor activation and downstream signaling, faster binding kinetics can contribute to enhanced signaling in ShHTL7 compared with AtD14. To test the effect of pocket entrance hydrophobicity, we suggest comparing GR24 binding for a V144T/A147S/A154S/F159T quadruple mutant AtD14 with wild-type $A t \mathrm{D} 14$ in an ITC assay. If pocket entrance anchoring of the ligand does indeed inhibit binding, the mutant should display higher binding affinity compared with the wild-type.

We also identified several key interactions involving residues on the D-loop of $A t \mathrm{D} 14$ that stabilize the D-loop in a catalytically inactive, D218-out conformation. While these interactions do not preclude binding, they likely hinder the catalytic process in AtD14 compared with in ShHTL7 by stabilizing catalytically inactive states of the protein. Assuming that the hydrolysis is an inducer of receptor activation and downstream signaling, the stabilization of catalytically inactive conformations of the protein can lead to decreased signaling as well. To test the effect of D-loop conformation on catalytic activity, we suggest comparing enzyme activity for a A216S/ K217N/S220M triple mutant AtD14 with wild-type AtD14 using a fluorescent strigolactone analog such as YLG (42). If these D-loop residues are important in modulating catalytic activity, the triple mutant would show higher enzymatic activity than wild-type $A t \mathrm{D} 14$.

Finally, we evaluated the hypothesis that a larger binding pocket in ShHTL7 enables its unique sensitivity to strigolactone. The average binding pocket volumes of AtD14 and ShHTL7 are nearly identical, however, AtD14 is able to access more low-volume states, which preclude ligand binding as well as more high-volume states, which allow more nonproductive binding to occur. These effects play a dual role in modulating ligand selectivity: ShHTL7 is less likely to adopt low-volume states, which are unable to bind ligand as well as highvolume states that are likely to bind ligand in nonproductive, signaling-inactive poses. To test the effect of pocket fluctuations on binding, we suggest comparing binding of GR24 to an E138Q mutant and a V144T/F159T/F195C triple mutant of $A t \mathrm{D} 14$ to its wild type in an ITC assay. These are both mutants that our simulations predict will stabilize low-volume states of $A t \mathrm{D} 14$. If these residues stabilize low-volume states and lowvolume states prevent binding, the mutants should show higher binding compared with wild-type AtD14.

A 10,000-fold difference in EC50 for downstream signaling implies a difference of $\sim 5.5-6 \mathrm{kcal} / \mathrm{mol}$ along the strigolactone signaling process. In total, combined effects of enhanced binding and more stable catalytically active state contribute $\sim 2-3 \mathrm{kcal} / \mathrm{mol}$. This indicates that while the differences in the ligand-binding process are contributors to the unique sensitivity of ShHTL7, subsequent steps in the signaling process, such as hydrolysis, activation, and association with signaling partners, play important roles in modulating this selectivity as well.
To our knowledge, this is the first in-depth characterization of the ligand-binding process in strigolactone receptors. This study demonstrates the utility of molecular simulations approaches in providing mechanistic insights into fundamental questions in the field of plant biology $(43,44)$. Due to the importance of strigolactone signaling in crop productivity and parasitic weed germination, there is great interest in developing strigolactone signalling antagonists (45-48). The factors we have identified that modulate ligand selectivity in strigolactone receptors can be used to inform the design of selective signalling agonists to enhance shoot branching in crops, induce suicidal germination in parasitic weeds, or prevent parasitic weed germination.

\section{Experimental procedures \\ Molecular dynamics simulations}

MD simulations were prepared using AmberTools 14/18 and run using Amber 14/18 (49). The protein was described using the ff14SB force field and water was described using the TIP3P model (50). The GR24 ligand was described using the generalized AMBER force field (GAFF) (51). Force field parameters for GR24 were generated using Antechamber. Initial structures for AtD14 and ShHTL7 were obtained from Protein Data Bank entries 4IH4 (52) and 5Z7Y (16), respectively. The GR24 substrate was superimposed into the binding pocket by structural alignment of the bound structure of OsD14 bound to GR24 (PDB 5DJ5) (18). For ShHTL7, an additional system was prepared with the ligand randomly placed in solution using Packmol (53). Additional details of our sampling protocol are listed in Tables S3 and S4. Each protein-ligand complex was solvated in a TIP3P water box of size $\sim 70 \times$ $70 \times 70 \AA . \mathrm{NaCl}$ was added at a concentration of $0.15 \mathrm{M}$ to neutralize the system. Each structure was minimized for 10,000 steps using the conjugate gradient descent method and equilibrated for $10 \mathrm{~ns}$. Production runs were performed for an aggregate of $207 \mu$ s for ShHTL7 and $198 \mu$ sor AtD14. Temperatures were held constant at $300 \mathrm{~K}$ using the Berendsen thermostat, and pressures were held constant at 1.0 bar using the Berendsen barostat. Full electrostatics were computed using the Particle Mesh Ewald algorithm with a cutoff distance of $10 \AA$ (54). Bonds to hydrogen were constrained using the SHAKE algorithm (55).

\section{Markov state model construction}

MSMs were constructed using the PyEmma (56) package. Thirty-one input distance features were computed using MDTraj 1.9.0 (57) (Table S5). The input distances were projected onto a reduced set of coordinates using time-lagged independent component analysis (TICA) (58, 59). The dimensionality-reduced coordinates were then clustered into states using the Mini-Batch K-Means algorithm prior to MSM estimation. The hyperparameters (number of TICA dimensions and number of clusters) were chosen via maximization of cross-validation scores (Fig. S6) (60). Lag time was chosen by convergence implied timescales with respect to lag time (Fig. S5). Final parameters for MSM construction are 


\section{Strigolactone perception in parasitic plants}

listed in Table S6. Markovianity of the model was further validated using the Chapman-Kolmogorov test (Fig. S8). Free energy landscapes were calculated by computing the probability distribution along chosen sets of order parameters $(x, y)$ and weighting each point by the equilibrium probability of its associated MSM state (Equation 1).

$$
F(x, y)=-R T \ln \left[P_{\text {raw }}(x, y) * \pi_{i}(x, y)\right]
$$

\section{Standard binding free energy calculation}

Standard free energies of binding were calculated using the volume correction method as detailed in Buch et al. (27). Briefly, this method corrects for nonstandard ligand concentration in the simulation by introducing a correction term (Equation 2) that corresponds to the free energy of moving the ligand from a $1 \mathrm{M}$ solution to simulation conditions. For calculation of bound state volume, the bound state was defined as points within $1.0 \mathrm{~nm}$ and $4.0 \mathrm{kcal} / \mathrm{mol}$ of the minimum free energy point on the three-dimensional MSM-weighted free energy landscape projected onto ligand position. Convergence of $\Delta G_{0}$ with respect to bound state definition is shown in Figure S10.

\section{Binding pocket volume calculation}

Binding pocket volumes were calculated using the POVME 2.0 package (61). For each protein, a "maximum englobing region" was defined as a sphere centered at the midpoint between the geometric center of the T1 and T2 helix C- $\alpha$ atoms (residues 138-165 for AtD14, residues 136-163 for ShHTL7) and the $\mathrm{C}-\alpha$ atom of the catalytic serine (Fig. S11). The radius of the maximum englobbing sphere was set as the distance between the center and the C- $\alpha$ of the catalytic serine. The probability distribution of binding pocket volumes was weighted by MSM equilibrium probability. The average and standard deviation of the pocket volumes were calculated using the MSM-weighted probability distributions.

$$
\Delta G_{0}=-R T \ln \frac{V_{b}}{V_{0}}-\Delta W
$$

\section{Residue-ligand contact probability calculation}

Residue-ligand distances for each residue were computed using MDTraj 1.9.0 (57). Contacts were defined as residueligand distances within a cutoff distance of $4.0 \AA$. The equilibrium contact probability was calculated as the product of raw contact probability within each MSM state multiplied by the equilibrium probability of the MSM state as shown in Equation 3:

$$
P_{\text {contact, eq }}=\left.\sum_{i}^{N_{\text {states }}} P_{\text {contact, raw }}\right|_{i} * \pi_{i}
$$

\section{Data availability}

Data and in-house code can be found at the following GitHub repository: https://github.com/ShuklaGroup/ Strigolactone-Binding-JBC2021.

Supporting information-This article contains supporting information $(35,36)$.

Acknowledgments - This research was supported by the Blue Waters sustained-petascale computing project, which is funded by the National Science Foundation (OCI-0725070 and ACI-1238993) and the state of Illinois.

Author contributions-D. C. N. and D. S., conceptualization; J. C., data curation; J. C. and D. S., formal analysis; D. S., funding acquisition; J. C., methodology; D. S., project administration; D. S., resources; J. C., software; D. C. N. and D. S., supervision; J. C., visualization; J. C., writing-original draft; A.W., D. C. N., and D. S., writing-review and editing.

Funding and additional information-J. C. acknowledges support from the Samuel W. Parr Graduate Fellowship (Department of Chemical and Biomolecular Engineering, University of Illinois) and the National Institutes of Health Chemistry-Biology Interface Training Grant (T32-GM070421). D. S. acknowledges support from the Center for Advanced Study at University of Illinois at UrbanaChampaign.

Conflicts of interest-The authors declare that they have no conflicts of interest with the contents of this article.

Abbreviations-The abbreviations used are: MD, molecular dynamics; MSM, Markov state model.

\section{References}

1. Umehara, M., Hanada, A., Yoshida, S., Akiyama, K., Arite, T., TakedaKamiya, N., Magome, H., Kamiya, Y., Shirasu, K., Yoneyama, K., Kyozuka, J., and Yamaguchi, S. (2008) Inhibition of shoot branching by new terpenoid plant hormones. Nature 455, 195-200

2. Gomez-Roldan, V., Fermas, S., Brewer, P. B., Puech-Pagès, V., Dun, E. A., Pillot, J. P., Letisse, F., Matusova, R., Danoun, S., Portais, J. C., Bouwmeester, H., Bécard, G., Beveridge, C. A., Rameau, C., and Rochange, S. F. (2008) Strigolactone inhibition of shoot branching. Nature 455, 189-194.

3. Waters, M. T., Gutjahr, C., Bennett, T., and Nelson, D. C. (2017) Strigolactone signaling and evolution. Annu. Rev. Plant Biol. 68, 291-322

4. Lumba, S., Holbrook-Smith, D., and McCourt, P. (2017) The perception of strigolactones in vascular plants. Nat. Chem. Biol. 13, 599-606

5. Cook, C. E., Whichard, L. P., Turner, B., Wall, M. E., and Egley, G. H. (1966) Germination of witchweed (striga lutea lour.): Isolation and properties of a potent stimulant. Science 154, 1189-1190

6. Ejeta, G., and Gressel, J. (2007) Integrating New Technologies For Striga Control, World Scientific, Singapore

7. Yao, R., Ming, Z., Yan, L., Li, S., Wang, F., Ma, S., Yu, C., Yang, M., Chen, L., Chen, L., Li, Y., Yan, C., Miao, D., Sun, Z., Yan, J., et al. (2016) Dwarf14. is a non-canonical hormone receptor for strigolactone. Nature 536, 469473

8. de Saint Germain, A., Clavé, G., Badet-Denisot, M. A., Pillot, J. P., Cornu, D., Le Caer, J. P., Burger, M., Pelissier, F., Retailleau, P., Turnbull, C., Bonhomme, S., Chory, J., Rameau, C., and Boyer, F. D. (2016) An histidine covalent receptor and butenolide complex mediates strigolactone perception. Nat. Chem. Biol. 12, 787-794 
9. Yao, R., Wang, F., Ming, Z., Du, X., Chen, L., Wang, Y., Zhang, W., Deng, H., and Xie, D. (2017) ShHTL7 is a non-canonical receptor for strigolactones in root parasitic weeds. Cell Res. 27, 838-84.1

10. Yao, R., Wang, L., Li, Y., Chen, L., Li, S., Du, X., Wang, B., Yan, J., Li, J., and Xie, D. (2018) Rice DWARF14 acts as an unconventional hormone receptor for strigolactone. J. Exp. Bot. 69, 2355-2365

11. Jiang, L., Liu, X., Xiong, G., Liu, H., Chen, F., Wang, L., Meng, X., Liu, G., Yu, H., Yuan, Y., Yi, W., Zhao, L., Ma, H., He, Y., Wu, Z., et al. (2013) DWARF 53 acts as a repressor of strigolactone signalling in rice. Nature 504, 4.01-4.05

12. Seto, Y., Yasui, R., Kameoka, H., Tamiru, M., Cao, M., Terauchi, R., Sakurada, A., Hirano, R., Kisugi, T., Hanada, A., Umehara, M., Seo, E., Akiyama, K., Burke, J., Takeda-Kamiya, N., et al. (2019) Strigolactone perception and deactivation by a hydrolase receptor DWARF14. Nat. Commun. 10, 191

13. Shabek, N., Ticchiarelli, F., Mao, H., Hinds, T. R., Leyser, O., and Zheng, N. (2018) Structural plasticity of d3-d14 ubiquitin ligase in strigolactone signalling. Nature 563, 652-656

14. Toh, S., Holbrook-Smith, D., Stogios, P. J., Onopriyenko, O., Lumba, S., Tsuchiya, Y., Savchenko, A., and McCourt, P. (2015) Structure-function analysis identifies highly sensitive strigolactone receptors in striga. Science 350, 203-207

15. Conn, C. E., Bythell-Douglas, R., Neumann, D., Yoshida, S., Whittington, B., Westwood, J. H., Shirasu, K., Bond, C. S., Dyer, K. A., and Nelson, D. C. (2015) PLANT EVOLUTION. Convergent evolution of strigolactone perception enabled host detection in parasitic plants. Science 349, $540-$ 543

16. Xu, Y., Miyakawa, T., Nosaki, S., Nakamura, A., Lyu, Y., Nakamura, H., Ohto, U., Ishida, H., Shimizu, T., Asami, T., and Tanokura, M. (2018) Structural analysis of HTL and d14 proteins reveals the basis for ligand selectivity in striga. Nat. Commun. 9, 3947

17. Bürger, M., Mashiguchi, K., Lee, H. J., Nakano, M., Takemoto, K., Seto, Y., Yamaguchi, S., and Chory, J. (2019) Structural basis of karrikin and nonnatural strigolactone perception in physcomitrella patens. Cell Rep. 26, 855-865

18. Zhao, L. H., Zhou, X. E., Yi, W., Wu, Z., Liu, Y., Kang, Y., Hou, L., de Waal, P. W., Li, S., Jiang, Y., Scaffidi, A., Flematti, G. R., Smith, S. M., Lam, V. Q., Griffin, P. R., et al. (2015) Destabilization of strigolactone receptor DWARF14 by binding of ligand and e3-ligase signaling effector DWARF3. Cell Res. 25, 1219-1236

19. Carlsson, G. H., Hasse, D., Cardinale, F., Prandi, C., and Andersson, I. (2018) The elusive ligand complexes of the DWARF14 strigolactone receptor. J. Exp. Bot. 69, 2345-2354

20. Moffett, A. S., Bender, K. W., Huber, S. C., and Shukla, D. (2017) Molecular dynamics simulations reveal the conformational dynamics of Arabidopsis thaliana BRI1 and BAK1 receptor-like kinases. J. Biol. Chem. 292, 12643-12652

21. Moffett, A. S., Bender, K. W., Huber, S. C., and Shukla, D. (2017) Allosteric control of a plant receptor kinase through s-glutathionylation. Biophys. J. 113, 2354-2363

22. Aldukhi, F., Deb, A., Zhao, C., Moffett, A. S., and Shukla, D. (2019) Molecular mechanism of brassinosteroid perception by the plant growth receptor BRI1. J. Phys. Chem. B 124, 355-365

23. Lawrenz, M., Shukla, D., and Pande, V. S. (2015) Cloud computing approaches for prediction of ligand binding poses and pathways. Sci. Rep. 5 , 7918

24. Shukla, S., Zhao, C., and Shukla, D. (2019) Dewetting controls plant hormone perception and initiation of drought resistance signaling. Structure 27, 692-702.e3

25. Selvam, B., Yu, Y.-C., Chen, L.-Q., and Shukla, D. (2019) Molecular basis of the glucose transport mechanism in plants. ACS Cent. Sci.

26. Hu, F., Liu, X. T., Zhang, J. L., Zheng, Q. C., Eglitis, R. I., and Zhang, H. X. (2019) MD simulation investigation on the binding process of smokederived germination stimulants to its receptor. J. Chem. Inf. Model. 59, $1554-1562$

27. Buch, I., Giorgino, T., and De Fabritiis, G. (2011) Complete reconstruction of an enzyme-inhibitor binding process by molecular dynamics simulations. Proc. Natl. Acad. Sci. U. S. A. 108, 10184-10189
28. Shan, Y., Kim, E. T., Eastwood, M. P., Dror, R. O., Seeliger, M. A., and Shaw, D. E. (2011) How does a drug molecule find its target binding site? J. Am. Chem. Soc. 133, 9181-9183

29. Selvam, B., Shamsi, Z., and Shukla, D. (2018) Universality of the sodium ion binding mechanism in Class A G-protein-coupled receptors. Angew Chem Int. Ed. Engl. 57, 3048-3053

30. Shukla, D., Hernández, C. X., Weber, J. K., and Pande, V. S. (2015) Markov state models provide insights into dynamic modulation of protein function. Acc. Chem. Res. 48, 414-422

31. Shamsi, Z., Cheng, K. J., and Shukla, D. (2018) Reinforcement learning based adaptive sampling: Reaping rewards by exploring protein conformational landscapes. J. Phys. Chem. B 122, 8386-8395

32. Shamsi, Z., Moffett, A. S., and Shukla, D. (2017) Enhanced unbiased sampling of protein dynamics using evolutionary coupling information. Sci. Rep. 7, 12700-12713

33. Prinz, J. H., Wu, H., Sarich, M., Keller, B., Senne, M., Held, M., Chodera, J. D., Schütte, C., and Noé, F. (2011) Markov models of molecular kinetics: Generation and validation. J. Chem. Phys. 134, 174105

34. Husic, B. E., and Pande, V. S. (2018) Markov state models: From an art to a science. J. Am. Chem. Soc. 140, 2386-2396

35. Kagiyama, M., Hirano, Y., Mori, T., Kim, S. Y., Kyozuka, J., Seto, Y., Yamaguchi, S., and Hakoshima, T. (2013) Structures of d14 and d14l in the strigolactone and karrikin signaling pathways. Genes Cells 18, 147160

36. Hameed, U. S., et al. (2018) Structural basis for specific inhibition of the highly sensitive ShHTL7 receptor. EMBO Rep., e45619

37. Uraguchi, D., Kuwata, K., Hijikata, Y., Yamaguchi, R., Imaizumi, H., Am, S., Rakers, C., Mori, N., Akiyama, K., Irle, S., McCourt, P., Kinoshita, T., Ooi, T., and Tsuchiya, Y. (2018) A femtomolar-range suicide germination stimulant for the parasitic plant striga hermonthica. Science 362, 13011305

38. Ashkenazy, H., Abadi, S., Martz, E., Chay, O., Mayrose, I., Pupko, T., and Ben-Tal, N. (2016) ConSurf 2016: An improved methodology to estimate and visualize evolutionary conservation in macromolecules. Nucleic Acids Res. 44, W344-W350

39. Nakamura, H., Xue, Y. L., Miyakawa, T., Hou, F., Qin, H. M., Fukui, K., Shi, X., Ito, E., Ito, S., Park, S. H., Miyauchi, Y., Asano, A., Totsuka, N., Ueda, T., Tanokura, M., and Asami, T. (2013) Molecular mechanism of strigolactone perception by DWARF14. Nat. Commun. 4, 2613

40. Abe, S., Sado, A., Tanaka, K., Kisugi, T., Asami, K., Ota, S., Kim, H. I., Yoneyama, K., Xie, X., Ohnishi, T., Seto, Y., Yamaguchi, S., Akiyama, K., Yoneyama, K., and Nomura, T. (2014) Carlactone is converted to carlactonoic acid by MAX1 in Arabidopsis and its methyl ester can directly interact with AtD14 in vitro. Proc. Natl. Acad. Sci. U. S. A. 111, 1808418089

41. Xu, Y., Miyakawa, T., Nakamura, H., Nakamura, A., Imamura, Y., Asami, T., and Tanokura, M. (2016) Structural basis of unique ligand specificity of KAI2-like protein from parasitic weed striga hermonthica. Sci. Rep. 6 , 31386

42. Tsuchiya, Y., Yoshimura, M., Sato, Y., Kuwata, K., Toh, S., HolbrookSmith, D., Zhang, H., McCourt, P., Itami, K., Kinoshita, T., and Hagihara, S. (2015) Probing strigolactone receptors in striga hermonthica with fluorescence. Science 349, 864-868

43. Feng, J., Chen, J., Selvam, B., and Shukla, D. (2019) Computational microscopy: Revealing molecular mechanisms in plants using molecular dynamics simulations. Plant Cell 31. tpc.119.tt1219

44. Moffett, A. S., and Shukla, D. (2018) Using molecular simulation to explore the nanoscale dynamics of the plant kinome. Biochem. J. 475, 905-921

45. Holbrook-Smith, D., Toh, S., Tsuchiya, Y., and McCourt, P. (2016) Smallmolecule antagonists of germination of the parasitic plant striga hermonthica. Nat. Chem. Biol. 12, 724-729

46. Xiang, H., Yao, R., Quan, T., Wang, F., Chen, L., Du, X., Zhang, W., Deng, H., Xie, D., and Luo, T. (2017) Simple $\beta$-lactones are potent irreversible antagonists for strigolactone receptors. Cell Res. 27, 15251528

47. Takeuchi, J., et al. (2018) Rationally designed strigolactone analogs as antagonists of the d14 receptor. Plant Cell Physiol. 


\section{Strigolactone perception in parasitic plants}

48. Nakamura, H., Hirabayashi, K., Miyakawa, T., Kikuzato, K., Hu, W., Xu, Y., Jiang, K., Takahashi, I., Niiyama, R., Dohmae, N., Tanokura, M., and Asami, T. (2019) Triazole ureas covalently bind to strigolactone receptor and antagonize strigolactone responses. Mol. Plant 12, 44-58

49. Case, D. A., Ben-Shalom, I. Y., Brozell, S. R., Cerutti, D. S., Cheatham, T. E., III, Cruzeiro, V. W. D., Darden, T. A., Duke, R. E., Ghoreishi, D., Gilson, M. K., Gohlke, H., Goetz, A. W., Greene, D., Harris, R., Homeyer, N., et al. (2018) AMBER2018, University of California, San Francisco, CA

50. Jorgensen, W. L., Chandrasekhar, J., Madura, J. D., Impey, R. W., and Klein, M. L. (1983) Comparison of simple potential functions for simulating liquid water. J. Chem. Phys. 79, 926-935

51. Wang, J., Wolf, R. M., Caldwell, J. W., Kollman, P. A., and Case, D. A. (2004) Development and testing of a general amber force field. J. Comput. Chem. 25, 1157-1174.

52. Zhao, L. H., Zhou, X. E., Wu, Z. S., Yi, W., Xu, Y., Li, S., Xu, T. H., Liu, Y., Chen, R. Z., Kovach, A., Kang, Y., Hou, L., He, Y., Xie, C., Song, W., et al. (2013) Crystal structures of two phytohormone signal-transducing $\alpha / \beta$ hydrolases: karrikin-signaling KAI2 and strigolactone-signaling DWARF14. Cell Res. 23, 436-439

53. Martínez, L., Andrade, R., Birgin, E. G., and Martínez, J. M. (2009) PACKMOL: A package for building initial configurations for molecular dynamics simulations. J. Comput. Chem. 30, 2157-2164.

54. Darden, T., York, D., and Pedersen, L. (1993) Particle mesh Ewald: AnN.log(N) method for Ewald sums in large systems. J. Chem. Phys. 98, 10089-10092
55. Ryckaert, J.-P., Ciccotti, G., and Berendsen, H. J. C. (1977) Numerical integration of the cartesian equations of motion of a system with constraints: Molecular dynamics of n-alkanes. J. Comput. Phys. 23, 327-341

56. Scherer, M. K., Trendelkamp-Schroer, B., Paul, F., Pérez-Hernández, G., Hoffmann, M., Plattner, N., Wehmeyer, C., Prinz, J.-H., and Noé, F. (2015) PyEMMA 2: A software package for estimation, validation, and analysis of markov models. J. Chem. Theory Comput. 11, 55255542

57. McGibbon, R. T., Beauchamp, K. A., Harrigan, M. P., Klein, C., Swails, J. M., Hernández, C. X., Schwantes, C. R., Wang, L. P., Lane, T. J., and Pande, V. S. (2015) MDTraj: A modern open library for the analysis of molecular dynamics trajectories. Biophys. J. 109, 1528-1532

58. Schwantes, C. R., and Pande, V. S. (2013) Improvements in markov state model construction reveal many non-native interactions in the folding of nt19. J. Chem. Theory Comput. 9, 2000-2009

59. Schwantes, C. R., Shukla, D., and Pande, V. S. (2016) Markov state models and tica reveal a nonnative folding nucleus in simulations of nug2. Biophys. J. 110, 1716-1719

60. McGibbon, R. T., and Pande, V. S. (2015) Variational cross-validation of slow dynamical modes in molecular kinetics. J. Chem. Phys. 142, 124.105

61. Durrant, J. D., Votapka, L., Sørensen, J., and Amaro, R. E. (2014) POVME 2.0: An enhanced tool for determining pocket shape and volume characteristics. J. Chem. Theory Comput. 10, 5047-5056 